\title{
Involuntary outpatient commitment did not reduce hospital recidivism, though may for some patient subgroups
}

Swartz MS, Swanson JW, Wagner HR, et al. Can involuntary outpatient commitment reduce hospital recidivism?: Findings from a randomized trial with severely mentally ill individuals. Am J Psychiatry 1999 Dec;156:1968-75.

QUESTIONS: In patients with severe mental illness, is involuntary outpatient commitment effective in reducing rehospitalisations and is effectiveness dependent on a sustained exposure to court ordered treatment? For what clinical groups is involuntary commitment most effective?

\section{Design}

Randomised, \{allocation concealed*\}†, unblinded*, controlled trial with 1 year of follow up.

\section{Setting}

Piedmont region of North Carolina, USA.

\section{Patients}

264 involuntarily admitted patients (mean age 40 y, 50\% men) with a diagnosis of schizophrenia, schizoaffective disorder, or other psychotic disorder or major affective disorder of at least 1 year duration. Other inclusion criteria were substantive functional impairment in the activities of daily living, intensive treatment within the previous 2 years, and awaiting a period of court ordered outpatient commitment. Exclusion criteria were primary diagnosis of personality disorder, psychoactive substance use disorder, organic brain syndrome in the absence of a primary psychotic or mood disorder, or a recent serious act of violence involving injury or use of a weapon.

\section{Intervention}

129 patients were allocated to continue under their outpatient commitment orders and 135 were allocated to be released from their outpatient commitment by notifying the court. All patients received case management services plus additional outpatient treatment. Total length of commitment order was determined by clinical and legal criteria. Intensity of treatment was allowed to vary clinically.

\section{Main outcome measures}

Number of hospital admissions and total length of admissions.

\section{Main results}

Analysis was by intention to treat. Hospital outcomes for the control and outpatient commitment groups did not differ overall. Data were re-analysed to determine if duration of outpatient commitment ( $<180$ days $v \geqslant 180$ days) had an effect on hospital use. Sustained outpatient commitment $(\geqslant 180$ days) reduced mean admissions and total hospital days compared with patients in the control group (table). Sustained outpatient commitment was more effective in patients with nonaffective psychotic diagnoses than in those with affective diagnoses.

\section{Conclusion}

Involuntary outpatient commitment did not reduce hospital recidivism, though may for some patient subgroups.

*See glossary

$\dagger$ Information provided by the author.

\section{COMMENTARY}

Much of the evidence for the effectiveness of involuntary outpatient treatment in reducing rehospitalisation for people with serious mental disorders has come from population studies, quasi-experimental designs, or from studies with small samples. In this study, Swartz et al present the first findings from a combined randomised control and observational study designed to examine both the outcomes of involuntary outpatient commitment and the mechanisms by which these outcomes are achieved. The results support the claim that involuntary outpatient treatment can contribute to substantial reductions in the number of rehospitalisations and in the length of these admissions for patients with nonaffective psychoses. The more important clinical and mental health policy implications, however, come from the observational aspects of the study considering the length of the involuntary commitment order and the intensity of outpatient treatment on the rehospitalisation data. The reduction in hospital admissions is evident in these data only for psychotically disordered patients who received sustained outpatient commitment of over 180 days whereas shorter periods of commitment had no effect. In addition, Swartz et al present evidence that this reduction only occurs for patients receiving higher intensity treatment in their outpatient management.

The authors recognise the limitations of these latter findings because of possible bias introduced through nonrandom assignment of length of commitment and intensity of treatment. Consequently, their additional data, which are forthcoming, on the effect of involuntary outpatient commitment on the diverse range of outcomes measured in the study will be crucially important in substantiating the conclusion that when outpatient commitment is effective it operates only when it is sustained and combined with intensive treatment.

Because of the variability in the clinical and legal considerations with regard to involuntary outpatient treatment orders between different countries and jurisdictions, mental health policy makers need to be particularly cautious in applying these findings. Nor should these data be a replacement for sound ongoing local evaluation of the outcomes of involuntary outpatient treatment.

Richard O'Kearney, BA, Dip App Psych, MPsych University of New South Wales Sydney, Australia

Source of funding National Institute of Mental Health.

For correspondence: $\mathrm{Dr}$ M S Swartz, Box 3173 , Duke University

Medical Center,

Durham, NC 27710,

USA.Fax +1919681 7504.

Involuntary outpatient commitment (IOC) v hospital release (control) in severe mental illness $\ddagger$

\begin{tabular}{llll|l} 
IOC $<180$ & $\begin{array}{l}\text { IOC } \geqslant 180 \\
\text { days } \\
\text { days } \\
\text { Mean (SD) }\end{array}$ & $\begin{array}{l}\text { Control } \\
\text { Mean (SD) }\end{array}$ & $\begin{array}{l}\text { Mean difference } \\
(\mathbf{9 5 \%} \text { CI) } \neq \\
\geqslant 180 \text { days v control }\end{array}$ \\
\hline Total admission & $0.9(1.2)$ & $0.5(0.8)$ & $1.0(1.6)$ & $-0.6(-1.1$ to -0.1$)$ \\
\hline Total days in hospital & $37.7(61.4)$ & $7.5(15.9)$ & $27.9(51.1)$ & $-20.4(-35.4$ to -5.5$)$ \\
\hline
\end{tabular}

‡Numbers calculated from data in article. 